\title{
The Effect of Electromagnetic Attenuation on the Spatial Spectrum of the Geomagnetic Field
}

\author{
H. W. S. McQueen, ${ }^{*}$ L. M. HaYes, ${ }^{* *}$ and D. Conley** \\ *York University, Earth Sciences, \\ Downsview, Ontario, Canada \\ **Physics Department, University of Queensland, \\ Brisbane, Australia
}

(Received June 3, 1982; Revised February 23, 1983)

\begin{abstract}
A comparison of the mean energy densities in the different spatial harmonics of the geomagnetic field with the corresponding scale times derived from the secular variation reveals a correlation which may be explained by greater attenuation of faster varying harmonics due to the finite electrical conductivity between the source level in the core and the surface of the earth. The conductivity required for the mantle alone to produce this effect is an order of magnitude too high to allow the observed sudden onset of some events in the surface field. It is found that the correlation may equally well be explained by a layer of core material $\sim 10 \mathrm{~km}$ thick screening the principal motions responsible for the secular variation. Surface field events with rise times up to several years must then involve a disturbance of this layer or be transported across it as a hydromagnetic wave disturbance.
\end{abstract}

\section{Introduction}

When the spatial spectrum of the geomagnetic field is extrapolated to the level of the core-mantle boundary, ignoring any effect of conductivity, the relatively greater enhancement of the higher harmonics makes the spatial spectrum nearly white, that is, the energy density contribution from each spherical harmonic degree decreases much more slowly with degree than at the surface of the earth. This trend led ELPHIC and RUSSELL (1978) to define an "apparent source depth" for processes generating the field as the level at which the spatial spectrum would become white by such an extrapolation. They estimated this level to be $470 \mathrm{~km}$ inside the core, in agreement with an earlier result of ELSASSER (1941) obtained from modelling of the field by a set of dipoles distributed at random on a sphere inside the earth. This value would be significantly different if the effects on the time varying spatial harmonics of conductivity in the overlying core and mantle were included. Although an unambiguous calculation of such effects would require knowledge of the conductivity profile and the time spectrum

*Now at R.S.E.S., Australian National University, Canberra, Australia, 2601. 
of each harmonic, it is possible to invert the argument to obtain constraints on the conductivity structure above some level at which a white spatial spectrum of the field is postulated to exist, using a rough estimate of the time spectrum.

Records of the geomagnetic field are not yet long enough for a detailed analysis of frequencies present in most harmonics. YUKUTAKE (1979) studied the dipole component and found periodicities of 60-70, 400-600, and 8,000 years. However the higher degree harmonics have been less well known than the dipole in the past. The studies of MCDONALD and GUNST (1967) and BARRACLOUGH $(1976,1978)$ indicate that reliable models of the field and secular variation to degree six are not available for epochs prior to this century although some parts of the field were well mapped back to the sixteenth century and much effort has gone into collating and interpolating the old charts. Even in this century the accuracy is poor (see, e.g. MALIN, 1969) due to the sparse and irregular distribution of magnetic observatories, particularly over the oceans.

\section{A Correlation}

A rough estimate of dominant periodicities is available from the secular variation as follows. The mean energy density in a magnetic field $\boldsymbol{B}$ over a spherical surface $\Gamma$ of radius $r$ is given by

$$
\varepsilon=\frac{1}{4 \pi r^{2}} \int_{\Gamma} \frac{1}{2 \mu_{0}} \boldsymbol{B} \cdot \boldsymbol{B} \mathrm{d} S
$$

In the insulating region near the surface of the earth, the contribution to the mean energy density in the field from the harmonics of the $n$-th degree (features of length scale $\sim$ circumference $/ n$ ) at time $t$ is

$$
\varepsilon_{n}(r, t)=\left(\frac{d}{r}\right)^{2 n+4} \frac{(n+1)}{2 \mu_{0}} \sum_{m=0}^{n}\left\{\left(g_{n}^{m}\right)^{2}+\left(h_{n}^{m}\right)^{2}\right\}
$$

where $r$ is the geocentric radius, $d$ is the radius of the earth and $g_{n}^{m}(t)$ and $h_{n}^{m}(t)$ are the Gauss-Schmidt coefficients of the spherical harmonic expansion of the field (LowEs, 1974). A similar quantity is defined for the secular variation field

$$
\mathscr{V}_{n}(r, t)=\left(\frac{d}{r}\right)^{2 n+4} \frac{(n+1)}{2 \mu_{0}} \sum_{m=0}^{n}\left\{\left(\dot{g}_{n}^{m}\right)^{2}+\left(\dot{h}_{n}^{m}\right)^{2}\right\}
$$

and from these two quantities we can define scale times

$$
\tau_{n}=\left\{\varepsilon_{n} / \mathscr{V}_{n}\right\}^{1 / 2}
$$

The scale times have been used as rough estimators of the dominant periodicity in each harmonic (e.g. BoOKER, 1969). Their reliability is improved if $\varepsilon_{n}$ and $\mathscr{V}_{n}$ are first 
time averaged as will be done here but reliable time spectra of the energy densities would be more rigorous. Unfortunately the brief history of reliable field models even to degree six does not yet warrant this more rigorous approach.

In the conducting region of the mantle, the field can no longer be represented by the gradient of a scalar potential and the above expressions are not valid. In this region, the field may be represented (BACKUS, 1958) as the sum of a poloidal field $\boldsymbol{S}$ and a toroidal field $\boldsymbol{T}$ which are associated with scalar fields $S$ and $T$ by

$$
\boldsymbol{S}=\nabla x \nabla x(\boldsymbol{r} S) \text { and } \boldsymbol{T}=\nabla x(\boldsymbol{r} T)
$$

The toroidal field vanishes outside the conducting region and diffuses independently of the poloidal field if the conductivity is a function of radius only (SMYLIE, 1965), a common assumption for the deep mantle which will be retained here. Hence the energy densities defined above find their expression in the conducting region in terms of the spherical harmonic expansion of the poloidal scalar $S$ (see appendix). The effect of conductivity in modifying the time varying field passing through it is obtained by solving the induction equation for the radial dependence of the harmonic components of the poloidal scalar as described in the appendix.

If the energy density is extrapolated to the core-mantle boundary assuming only geometrical attenuation,

$$
\varepsilon_{n}^{g}(b)=\left(\frac{d}{b}\right)^{2 n+4} \varepsilon_{n}(d)
$$

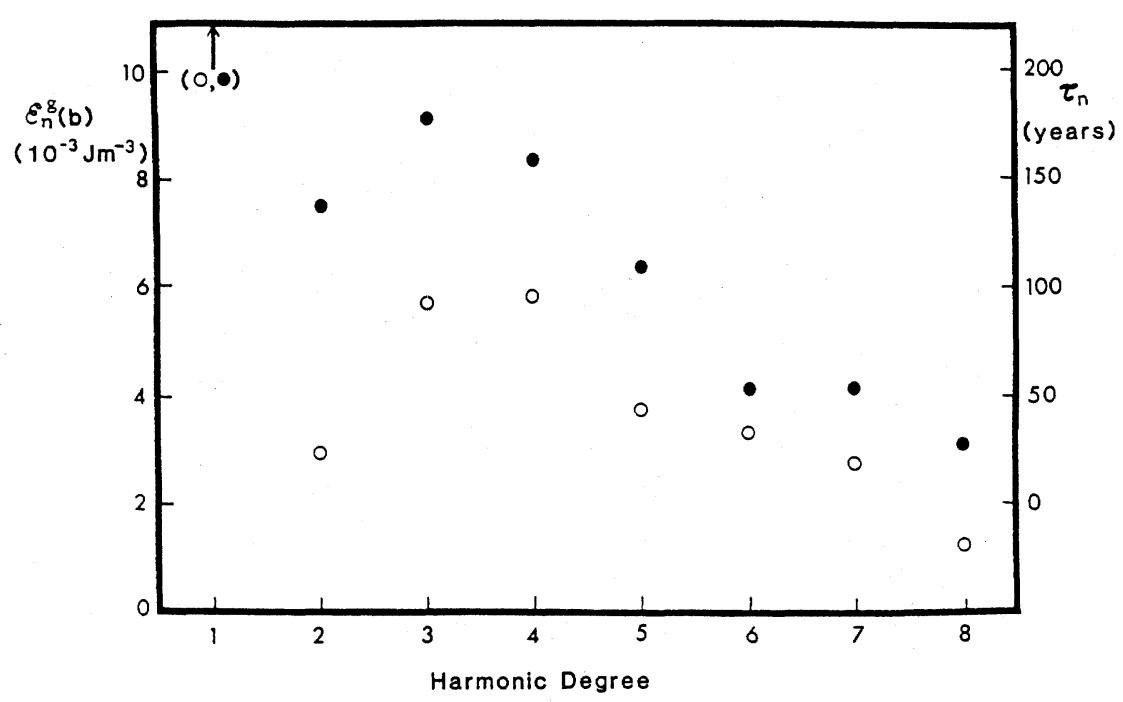

Fig. 1. Mean energy densities $(O)$ and scale times (O) as a function of harmonic degree in the geomagnetic field extrapolated to the surface of the core without allowance for intervening conductivity structure. Data from BARRAClOUGH et al. (1975). 
then any effects of electromagnetic attenuation should become more apparent. The plot of $\varepsilon_{n}^{g}$ and $\tau_{n}$ against $n$ from the model of BARRACLOUGH et al. (1975) shown in Fig. 1 displays a correlation between the geometrically corrected energy densities and the scale times whose sense, the weaker components of the field having shorter scale times, is compatible with the hypothesis that it is due to a greater electromagnetic attenuation of the more rapidly varying (usually smaller scale) components. This correlation is still present in a time averaged model covering the past 40 years derived from the models of Malin (1969), Malin and Clark (1974), HuRwitz et al. (1974), Peddie and Fabiano (1976), BARraClough et al. (1975), and BARRAClOUgH and BARKER (1980) (Figs. 2, 3). If we suppose that conduction in the mantle is responsible for this and that the energy densities in degrees 1 to 6 are approximately the same at core level, then we require a much higher conductivity than is normally quoted for the mantle (STACEY et al., 1978). This is because attenuation must be appreciable for field components with periods of tens to hundreds of years. This inference is clearly contradicted by the observed short rise time of the " 1970 jerk" reported by DuCRUIX et al. (1980) which disallows mantle conductivities much greater than about $100 \mathrm{~S} / \mathrm{m}$.

\section{Method}

In view of this it is necessary to look for an alternative explanation for the correlation. Numerical modelling (MCQUEEN, 1981) using the eigenvalue formulation developed by SMYLIE (1965) and JoHNSON and SMYLIE (1970) for the attenuation of a

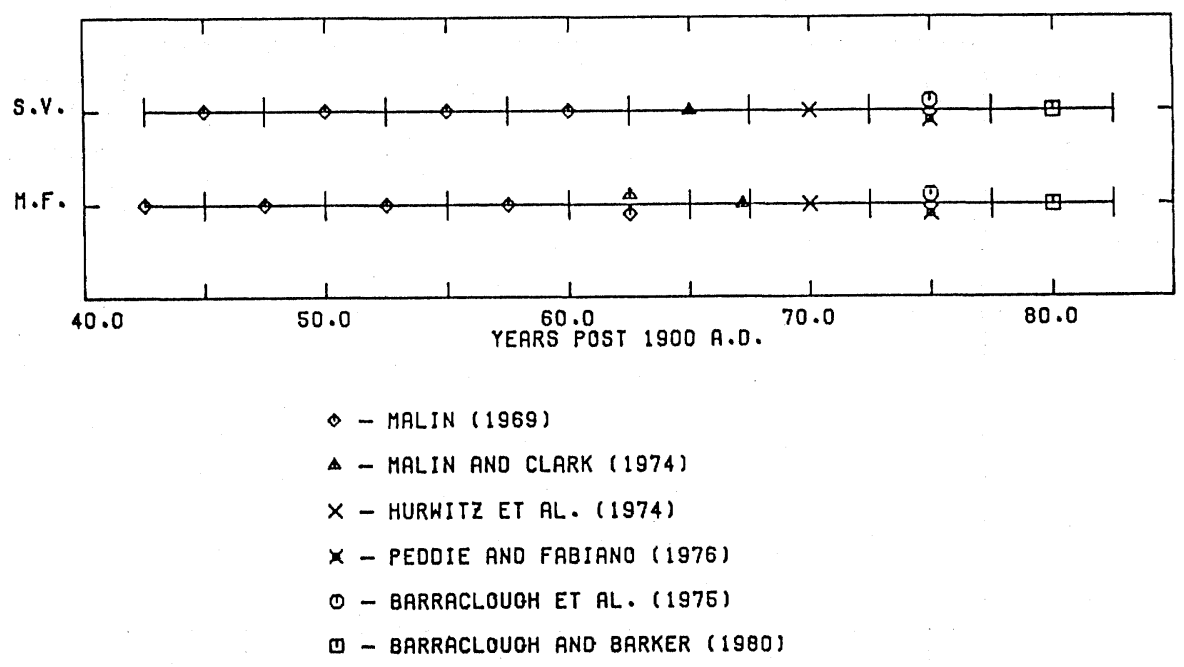

Fig. 2. Coverage of component models in the time averaged geomagnetic field model of Fig. 3. Models of the main field (M. F.) and secular variation (S. V.) are taken to represent the field in the intervals marked by vertical bars. Where two models appear in one interval, an equal weight average was used. 


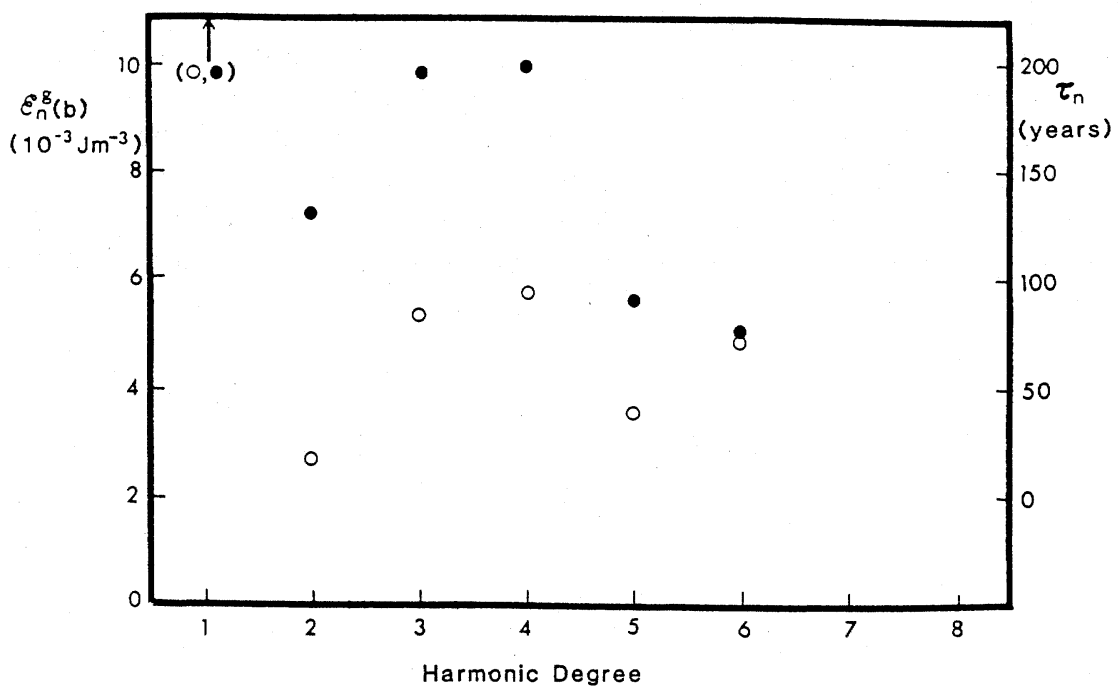

Fig. 3. As for Fig. 1 but using the time averaged values of $\varepsilon_{n}(d)$ and $\mathscr{V}_{n}(d)$ described in the text to obtain $\varepsilon_{n}^{g}(b)$ and $\tau_{n}$.

time varying magnetic field diffusing through a conducting spherical shell (see appendix) indicates that it is not possible to estimate both the conductivity and thickness of an attenuating layer from this kind of correlation given the present accuracy of time averaged energy densities and the unavoidable uncertainty of the exact nature of the core level time and spatial spectra. It is found that the single quantity best constrained is a moment of the conductivity distribution defined by

$$
K_{\beta}=\sigma L^{\beta}
$$

where $\sigma$ is the uniform conductivity required in a layer of thickness $L$ to minimise the scatter of the energy densities at $r=b$. The particular moment $(\beta)$ as well as its value $(K)$ are empirically determined from the required uniform conductivity values for various layer thicknesses and are dependent on the nature of the input time and spatial spectra.

As shown in the appendix, some assumption must be made about the form of the the core level time spectra in order to extrapolate the energy densities through the conducting layer. Moments have been estimated using the two extreme assumptions that the time spectra of the core level harmonics are:

a) monochromatic with a single characteristic frequency for each degree and

b) white up to a cutoff frequency which characterizes each degree.

Because the effect of a conducting layer on spatial harmonics with the same time spectrum is to relatively enhance the higher harmonics (MCDONALD, 1957), characteristic frequencies must increase with spatial degree to match the observed trend to decreasing energy in higher harmonics which persists after geometrical attenuation has 
been removed. An approach consistent with this is to infer the characteristic frequencies from the scale times. This is done by varying the characteristic frequency at core level until the observed scale times are reproduced at $r=d$.

Using the above time spectral assumptions, moments of the conductivity distribution responsible for the correlation are determined by iteratively minimizing the scatter $s(\sigma, L)$ of the mean energy densities in each degree at core level (whitening the spatial spectrum) using characteristic frequencies inferred from the scale times. The scatter is defined as

$$
s(\sigma, L)=\frac{\left[\sum_{n=2}^{N}\left(\bar{\varepsilon}_{n}(b)-E_{n}\right)^{2}\right]^{1 / 2}}{(N-2) E_{n}}
$$

where

$$
E_{n}=\frac{1}{N-1} \sum_{n=2}^{N} \bar{\varepsilon}_{n}(b)
$$

and the time averaged energy densities, $\bar{\varepsilon}_{n}$, at core level are related to those at the surface through the power transfer function $Q_{n}(\omega)$ derived in the appendix. $Q_{n}(\omega)$ is varied by changing $\sigma$ for fixed values of $L$ until the scatter reaches a minimum.

On this basis, the range of moments constrained by degrees two to six averaged over field models covering the past 40 years (Fig. 2) is

$$
\sigma L^{\beta}=(2.1 \pm 0.7) \times 10^{6} \mathrm{Sm}^{-1}(\mathrm{~km})^{\beta}
$$

where

$$
\beta=1.0 \pm 0.1
$$

That is, a layer thickness of $2,000 \mathrm{~km}$ requires a conductivity of $1,050 \mathrm{~S} / \mathrm{m}$ and any thinner layer would require higher conductivity. Figure 4 shows how required conductivities vary with assumed layer thicknesses when various time spectral assumptions and harmonic degree ranges are used.

\section{Discussion}

Since even the thickest mantle layer implies conductivity an order of magnitude higher than is consistent with the rise time of the 1970 jerk, it is tempting to conclude that most of this electromagnetic attenuation occurs in the outer layers of the core. For core conductivity $\left(3 \times 10^{5} \mathrm{~S} / \mathrm{m}\right)$ the required thickness is around $10 \mathrm{~km}$.

Two possible conclusions are:

1) The correlation does not reflect attenuation but arises from some as yet unknown feature of the dynamo mechanism or from an inadequate time base of observations. 


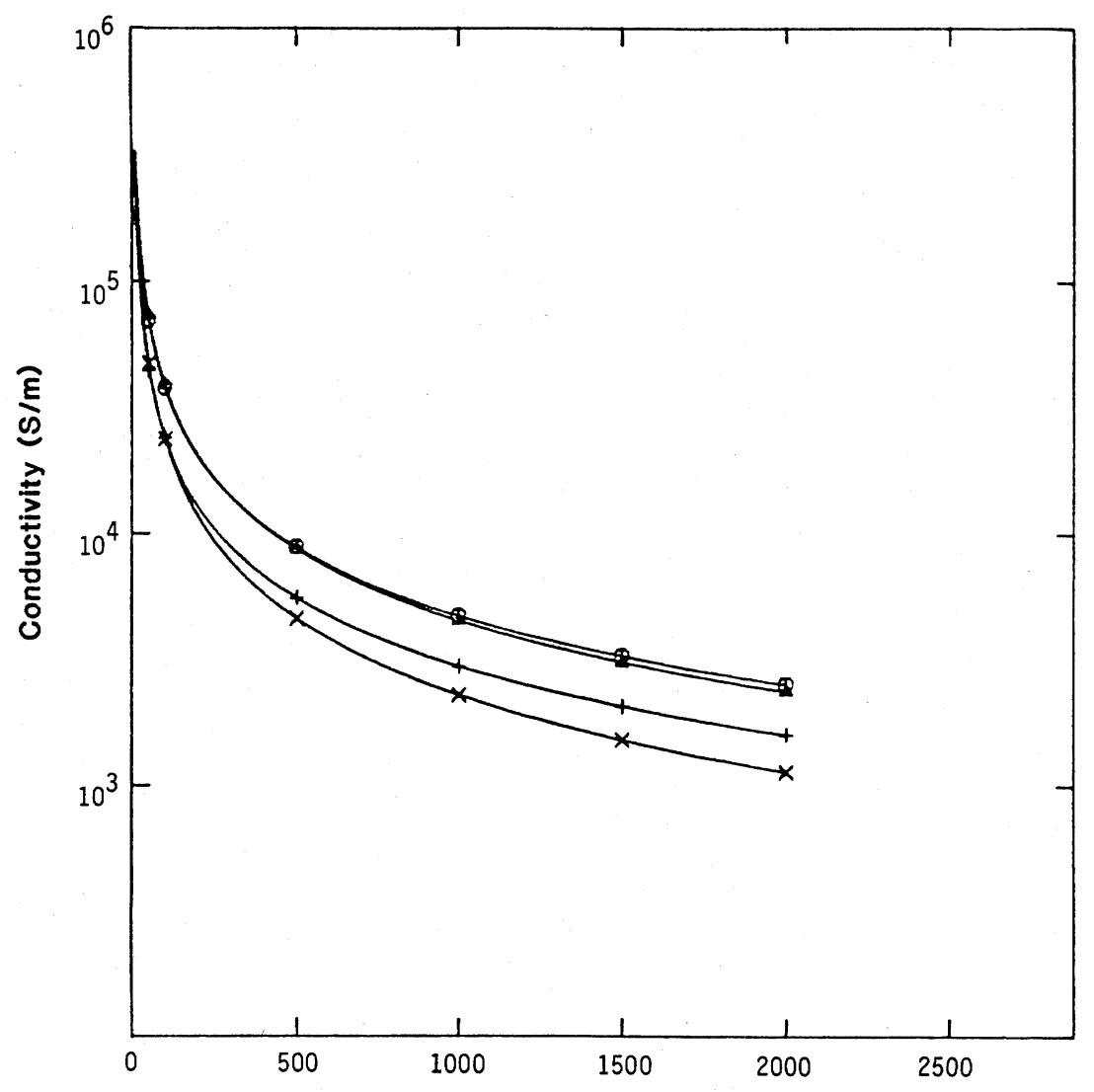

Layer Thickness $(\mathrm{km})$

Fig. 4. Conductivity as a function of layer thickness (for uniformly conducting layers) required to minimize the scatter of harmonic energy densities at core level. $\odot / \mathbf{\Delta}$, degrees 2 to 6 used, assuming monochromatic/white time spectrum in the core level field; $+/ \times$, degrees 2 to 5 used, assuming monochromatic/white time spectrum in the core level field.

2) Electromagnetic attenuation is occurring in a boundary layer at the surface of the core that does not normally participate in free stream motions. Such a boundary layer in which circulation was restricted to scales of the order of the layer thickness would appear electromagnetically "solid" to the large scale field variations of degree 1 to 8 (wavelength $>2,000 \mathrm{~km}$ ) in the sense that diffusion would dominate over convection of the field at that wavelength in the boundary layer.

If the second alternative is considered, an explanation must be found for the short rise time of the 1970 jerk since diffusion of the signal through such a boundary layer would produce a much slower rise time. The correspondence of field jerks with minima 
in the earth's rotation rate, pointed out by DucRuix et al. (1980), marks these as exceptional events caused perhaps by unusually vigorous core motions penetrating the boundary layer. Alternatively, SKILES (1972) has shown that the transmission of electromagnetic waves across the core-mantle boundary is dependent on the local field orientation and material properties. The appearance of the jerk may result from a strong disturbance encountering the core-mantle boundary in a region particularly favourable to transmission. Either of these mechanisms is consistent with the presence of a boundary layer partially screening all but a few exceptional events in the core from the surface.

WHALER (1980) and GUBBINs et al. (1982) have indicated independent evidence for a non-convecting layer at the top of the core. Certainly such a layer could not be much thicker than a few tens of kilometres without requiring sharply increasing energy density in higher multipoles of the field. Higher order harmonic expansions, particularly of the secular variation field, over a longer timespan may allow further conductivity information to be obtained from the geomagnetic spatial spectrum.

The authors express their appreciation for advice and discussions with F. D. Stacey, D. E. Smylie, and M. G. Rochester.

\section{APPENDIX}

The diffusion problem

The diffusion of the poloidal field is characterized by an equation governing the radial coefficients $s_{n}^{m}$ in the spherical harmonic expansion of the poloidal scalar:

$$
S=\sum_{n \geqslant 1} \sum_{m=-n}^{n} S_{n}^{m}(r, t) P_{n}^{m}(\cos \theta) \mathrm{e}^{i m \phi}
$$

where $r, \theta, \phi$ are the usual spherical polar coordinates representing radius, colatitude, and east longitude and the $P_{n}^{m}$ are (un-normalized) associated Legendre functions defined by

$$
P_{n}^{m}(\mu)=(-)^{m} \frac{\left(1-\mu^{2}\right)^{m / 2}}{2^{n} n !} \frac{d^{n+m}}{d \mu^{n+m}}\left(\mu^{2}-1\right)^{n} \text { for } m \geqslant 0
$$

and

$$
P_{n}^{-m}(\mu)=(-)^{m} \frac{(n-m) !}{(n+m) !} P_{n}^{m}(\mu) .
$$

Since the field $\boldsymbol{S}$ must be real

$$
s_{n}^{-m}(r, t)=(-)^{m} \frac{(n+m) !}{(n-m) !} s_{n}^{m *}(r, t)
$$


where the asterisk denotes complex conjugation. Using this expansion the vector structure of $S$ is

$$
\begin{aligned}
\left(\boldsymbol{S}_{n}^{m}\right)_{r} & =\frac{n(n+1)}{r} S_{n}^{m} P_{n}^{m}(\cos \theta) \mathrm{e}^{i m \phi} \\
\left(\boldsymbol{S}_{n}^{m}\right)_{\theta} & =\frac{1}{r} \frac{\partial}{\partial r}\left(r S_{n}^{m}\right) \frac{\mathrm{d} P_{n}^{m}(\cos \theta)}{\mathrm{d} \theta} \mathrm{e}^{i m \phi} \\
\left(\boldsymbol{S}_{n}^{m}\right)_{\phi} & =\frac{i m}{r \sin \theta} \frac{\partial}{\partial r}\left(r S_{n}^{m}\right) P_{n}^{m}(\cos \theta) \mathrm{e}^{i m \phi}
\end{aligned}
$$

and it is apparent that the diffusion of the radial vector component of the field is governed by the "radial coefficient" $s_{n}^{m}(r, t)$ while the two transverse components are governed by the "transverse coefficient"

$$
s_{n}^{m \dagger}(r, t)=\frac{\partial}{\partial r}\left(r s_{n}^{m}(r, t)\right)
$$

Application of the orthogonality relations for associated Legendre functions to the induction equation governing the diffusion of a magnetic field in a conducting medium leads to the equation governing the radial diffusion of the $s_{n}^{m}$ (SMYLIE, 1965)

$$
\frac{1}{r} \frac{\partial^{2}}{\partial r^{2}}\left(r s_{n}^{m}\right)-\frac{n(n+1)}{r^{2}} s_{n}^{m}=\mu_{0} \sigma \frac{\partial s_{n}^{m}}{\partial t} .
$$

The $s_{n}^{m}$ therefore describe a scalar field which diffuses through a spherical shell of diffusivity $\left(\mu_{0} \sigma\right)^{-1}$. Outside the conducting region, assumed to be a shell of outer radius $r=c, \mathrm{~A} .2$ becomes homogeneous and in the absence of external sources we have

$$
\begin{aligned}
s_{n}^{m}(r, t) & =\left(\frac{d}{r}\right)^{n+1} s_{n}^{m}(d, t) \\
s_{n}^{m \dagger}(r, t) & =-n s_{n}^{m}(r, t)=\left(\frac{d}{r}\right)^{n+1} s_{n}^{m \dagger}(d, t) .
\end{aligned}
$$

While the field observed at $r=d$ contains a small external contribution, the internal and external parts of the field can be separated since their different radial dependences imply different radial to transverse component ratios.

In the conducting region, A.2 may be Fourier transformed to

$$
r^{2} \frac{\partial^{2} \tilde{s}_{n}^{m}}{\partial r^{2}}+2 r \frac{\partial \tilde{s}_{n}^{m}}{\partial r}+\left[\alpha^{2} r^{2}-n(n+1)\right] s_{n}^{m}=0
$$

where 


$$
\tilde{s}_{n}^{m}(r, \omega)=\int_{-\infty}^{\infty} s_{n}^{m}(r, t) \mathrm{e}^{-i \omega t} \mathrm{~d} t
$$

and

$$
\alpha^{2}=-\mu_{0} \sigma i \omega
$$

subject to the boundary conditions A.3 at $r=c$. SMYLIE (1965) and JoHNSON and SMYLIE (1970) have shown that a solution of A.4 is given by

$$
\tilde{S}_{n}^{m}(r, \omega)=\left(\frac{d}{c}\right)^{n+1}\left(\frac{c}{r}\right) M_{n}(r, i \omega) \tilde{S}_{n}^{m}(d, \omega)
$$

and that $M_{n}(b, i \omega)$ can be represented as the infinite product

$$
M_{n}(b, i \omega)=\left(\frac{c}{b}\right)^{n} \prod_{j \geqslant 1}\left(1+i \omega / \lambda_{n j}\right)
$$

where the $\lambda_{n j}$ are the real positive eigenvalues of the Sturm-Liouville problem

$$
\begin{aligned}
& \frac{\mathrm{d}^{2} M}{\mathrm{~d} r^{2}}-\frac{n(n+1)}{r^{2}} M_{n}+\mu_{0} \sigma(r) \lambda M_{n}=0 \\
& M_{n}(c, \lambda)=1 \\
& \left(\frac{\mathrm{d} M_{n}(r, \lambda)}{\mathrm{d} r}\right)_{r=c}=-\frac{n}{c} \\
& M_{n}\left(b, \lambda_{n j}\right)=0 .
\end{aligned}
$$

This problem many be solved numerically for any form of conductivity profile and the eigenvalues stored for later use. Moreover, inspection of the differential system A.6 shows that scaling up the conductivity by a constant factor results in scaling down the eigenvalues by the same factor so that the conductivity profile may be scaled as desired with little extra computational effort. That the same may be said for a change in frequency is apparent from A.5.

The analogous solution for the transverse coefficient is

$$
\tilde{s}_{n}^{m \dagger}(r, \omega)=\left(\frac{d}{c}\right)^{n+1}\left(\frac{-c}{n}\right) \frac{\partial M_{n}(r, i \omega)}{\partial r} \tilde{s}_{n}^{m \dagger}(d, \omega)
$$

where

$$
\left[\frac{\partial M_{n}(r, i \omega)}{\partial r}\right]_{r=b}=\left(\frac{c}{b}\right)^{n}\left(\frac{-n}{b}\right) \prod_{j \geqslant 1}\left(1+i \omega / \lambda_{n j}^{\dagger}\right)
$$


and the $\lambda_{n j}^{\dagger}$ are the real positive eigenvalues of A.6 when A.7 is replaced by

$$
\left[\frac{\partial}{\partial r} M_{n}\left(r, \lambda_{n j}^{\dagger}\right)\right]_{r=b}=0
$$

The whole mantle system functions in this formulation are therefore

$$
P_{n}^{(\dagger)}(\omega)=\left(\frac{b}{d}\right)^{n+1} \prod_{j \geqslant 1}\left(1+i \omega / \lambda_{n j}^{(\dagger)}\right)^{-1}
$$

where the bracketed dagger denotes that two equations hold, one with and one without daggers, for the transverse and radial components respectively. Comparison with A.3 shows that the effect of conductivity in attenuating the time varying field is contained in the product terms in A.8 separately from the geometrical attenuation $(b / d)^{n+1}$. This conductive attenuation displays the character of a first order low pass Butterworth filter (KANASEWICH, 1975).

\section{Spatial spectra}

The gross spatial structure of the field at any radius may be characterised by the mean energy over a spherical surface at that radius, defined by

$$
\varepsilon(r, t)=\frac{1}{4 \pi} \int_{0}^{\pi} \mathrm{d} \theta \int_{0}^{2 \pi} \mathrm{d} \phi \frac{1}{2 \mu_{0}}|\boldsymbol{S}(r, \theta, \phi, t)|^{2} \sin \theta .
$$

Using the orthogonality properties (SMYLIE, 1965), this may be written as

$$
\varepsilon(r, t)=\sum_{n \geqslant 1} \varepsilon_{n}(r, t)
$$

where

$$
\varepsilon_{n}(r, t)=\frac{n(n+1)}{2 \mu_{0}(2 n+1)} \sum_{m=-n}^{n} \frac{(n+m) !}{(n-m) !}\left[\frac{n(n+1)}{r^{2}}\left|s_{n}^{m}\right|^{2}+\frac{1}{r^{2}}\left|s_{n}^{m \dagger}\right|^{2}\right]
$$

For each harmonic degree $n$, the quantity $\varepsilon_{n}(r, t)$ represents the total contribution to the mean energy density from features in the poloidal field of wavelength $2 \pi r / n$ at time $t$ and a plot of $\varepsilon_{n}$ versus $n$ provides a discrete spatial spectrum of the field (LowES, 1974). Replacing $S$ by the time derivative $S$ in A.9 defines the quantity $\mathscr{V}(r, t)$ which characterizes the gross structure of the secular variation field in the same way:

$$
\begin{aligned}
& \mathscr{V}(r, t)=\sum_{n \geqslant 1} \mathscr{V}_{n}(r, t) \\
& \mathscr{V}_{n}(r, t)=\frac{n(n+1)}{2 \mu_{0}(2 n+1)} \sum_{m=-n}^{n} \frac{(n+m) !}{(n-m) !}\left[\frac{n(n+1)}{r^{2}}\left|\dot{s}_{n}^{m}\right|^{2}+\frac{1}{r^{2}}\left|s_{n}^{m \dagger}\right|^{2}\right] .
\end{aligned}
$$


A relation derived by Rochester and SMYLIE (1965) between the $s_{n}^{m}$ and the conventional Gauss-Schmidt coefficients can be used to show the equivalence between (A.10, A.11) and $(1,2)$ in the text.

Power transfer function for the mantle

Generalising the representation of the geomagnetic field in terms of energy densities, a surface mean autocorrelation estimator for a finite duration sample of the vector field $S$ is defined by

$$
\tilde{\Phi}_{\mathrm{SS}}(r, \tau)=\frac{1}{8 \pi \mu_{0} r^{2}} \oiint_{\Gamma}\left[\frac{1}{T} \int_{-T / 2}^{T / 2} \boldsymbol{S}(r, \theta, \phi, t) \cdot \boldsymbol{S}(r, \theta, \phi, t-\tau) \mathrm{d} t\right] \mathrm{d} A
$$

where $\Gamma$ is a spherical surface of radius $r$ around the geocentre and $T$ is the duration of the sample. Then

$$
\tilde{\Phi}_{\mathrm{SS}}(r, 0)=\bar{\varepsilon}(r)=\frac{1}{T} \int_{-T / 2}^{T / 2} \varepsilon(r, t) \mathrm{d} t
$$

where a superposed bar indicates a time average over the duration $T$ and $\tilde{\Phi}$ indicates a sample autocorrelation. By analogy with the Wiener-Khintchine theorem from time sequence analysis, the Fourier transform of the autocorrelation is the power spectral density of the field at radius $r$ :

$$
\mathscr{S}_{\mathrm{SS}}(r, \omega)=\int_{-\infty}^{\infty} \Phi_{\mathrm{SS}}(r, \tau) \mathrm{e}^{-i \omega \tau} \mathrm{d} \tau
$$

and

$$
\bar{\varepsilon}(r)=\int_{-\infty}^{\infty} \mathscr{S}_{\mathrm{SS}}(r, \omega) \mathrm{d} f .
$$

We may also apply this development to the time derivative of the field replacing $S$ by $S$ deducing that

$$
\overline{\mathscr{V}}(r)=\int_{-\infty}^{\infty} \mathscr{S}_{\dot{\mathrm{s}} \mathbf{\mathrm { S }}}(r, \omega) \mathrm{d} f
$$

and define the scale times

$$
\bar{\tau}_{n}(r)=\left[\bar{\varepsilon}_{n}(r) / \overline{\mathscr{V}}_{n}(r)\right]^{1 / 2}
$$

Using A.1 and the orthogonality relations from SMYLIE (1965), we may write 


$$
\Phi_{\mathrm{SS}}(r, \tau)=\sum_{n \geqslant 1} \frac{n(n+1)}{r^{2}(2 n+1)} \sum_{m=-n}^{n} \frac{(n+m) !}{(n-m) !}\left[n(n+1) \psi_{n}^{m}(r, \tau)+\psi_{n}^{m \dagger}(r, \tau)\right]
$$

where

$$
\psi_{n}^{m(\dagger)}(r, \tau)=\frac{1}{T} \int_{-T / 2}^{T / 2} s_{n}^{m(\dagger)}(r, t) s_{n}^{m(\dagger) *}(r, t-\tau) \mathrm{d} t
$$

Fourier transforming the autocorrelation using this result gives

$$
\mathscr{S}_{\mathrm{SS}}(r, \omega)=\sum_{n \geqslant 1} \frac{n(n+1)}{r^{2}(2 n+1)} \sum_{m=-n}^{n} \frac{(n+m) !}{(n-m) !}\left[n(n+1) \zeta_{n}^{m}(r, \omega)+\zeta_{n}^{m \dagger}(r, \omega)\right](\mathrm{A} .13)
$$

where

$$
\zeta_{n}^{m(\dagger)}(r, \omega)=\int_{-\infty}^{\infty} \psi_{n}^{m(\dagger)}(r, \tau) \mathrm{e}^{-i \omega \tau} \mathrm{d} \tau
$$

are the power spectral densities in the radial and transverse coefficients respectively. A standard result from time sequence analysis (e.g. KANASEWICH, 1975, p86) permits the representation of these spectra at $r=d$ in terms of their values at $r=b$ and the system functions from A.8 as

$$
\begin{aligned}
\zeta_{n}^{m(\dagger)}(d, \omega) & =\left|P_{n}^{(\dagger)}(\omega)\right|^{2} \zeta_{n}^{m(\dagger)}(b, \omega) \\
& =\left(\frac{b}{d}\right)^{2 n+2} \prod_{j \geqslant 1}\left(1+\omega^{2} / \lambda_{m j}^{(\dagger) 2}\right)^{-1} \zeta_{n}^{m(\dagger)}(b, \omega)
\end{aligned}
$$

for real $\omega$. By virtue of A.3b we have also

$$
\zeta_{n}^{m \dagger}(d, \omega)=n^{2} \zeta_{n}^{m}(d, \omega)
$$

These last two results may be used to evaluate A.13 at $r=b$ and $r=d$ in terms of $\zeta_{n}^{m}(d, \omega)$. Comparison then shows that if we write

$$
\mathscr{S}_{\mathrm{SS}}(r, \omega)=\sum_{n \geqslant 1} \mathscr{S}_{\mathrm{SS}, n}(r, \omega)
$$

then

$$
\mathscr{S}_{\mathrm{SS}, n}(d, \omega)=Q_{n}(\omega) \mathscr{S}_{\mathrm{SS}, n}(b, \omega)
$$

where 


$$
Q_{n}(\omega)=\left(\frac{b}{d}\right)^{2 n+4}(2 n+1)\left[(n+1) \prod_{j \geqslant 1}\left(1+\omega^{2} / \lambda_{n j}^{2}\right)+n \prod_{j \geqslant 1}\left(1+\omega^{2} / \lambda_{n j}^{\dagger 2}\right)\right]^{-1}
$$

is the mantle power transfer function for the complete magnetic field. Finally, applying this to A.12,

$$
\bar{\varepsilon}_{n}(d)=\int_{-\infty}^{\infty} Q_{n}(\omega) \mathscr{S}_{\mathrm{SS}, n}(b, \omega) \mathrm{d} f
$$

Energy densities at core level, given by A.12 evaluated at $r=b$, are thus related to energy densities at the surface through the power transfer function $Q_{n}(\omega)$ which contains the effects of the conductive and geometric attenuation in the mantle. Note that in order to extrapolate the energy densities to core level, some assumption must be made about the form of the core level time spectrum $\mathscr{S}_{\mathrm{SS}, n}$.

\section{REFERENCES}

BaCkUs, G., A class of self-sustaining dissipative spherical dynamos, Ann. Phys., 4, 372-447, 1958.

BARRAClough, D. R., Spherical harmonic analysis of the geomagnetic secular variation-a review of methods, Phys. Earth Planet. Inter., 12, 365-382, 1976.

BarraClough, D. R., Spherical harmonic models of the geomagnetic field, Geomagn. Bull. Inst. Geol. Sci., No. 8, 1978.

Barraclough, D. R. and F. S. Barker, Reported in EOS, Trans. Am. Geophys. Union, 61, 453, 1980.

Barraclough, D. R., J. M. Harwood, B. R. Leaton, and S. R. C. Malin, A model of the geomagnetic field at epoch 1975, Geophys. J. R. Astr. Soc., 43, 645-659, 1975.

Booker, J. R., Geomagnetic data and core motions, Proc. R. Soc. Lond. A, 309, 27-40, 1969.

DuCruix, J., V. Courtillot, and J.-L LE MOUEL, The late 1960's secular variation impulse, the eleven year magnetic variation and the electrical conductivity of the deep mantle, Geophys. J. R. Astr. Soc., 61, 73-94, 1980.

ELPHIC, R. C. and C. T. RusSELL, On the apparent source depth of planetary magnetic fields, Geophys. Res. Lett., 5, 211-214, 1978.

Elsasser, W. M., A statistical analysis of the earth's internal magnetic field, Phys. Rev., 60, 876-883, 1941.

Elsasser, W. M., Hydromagnetic dynamo theory, Rev. Mod. Phys., 22, 1-35, 1956.

Gubbins, D., C. J. Thomson, and K. A. WhaleR, Stable regions in the earth's liquid core, Geophys. J. R. Astr. Soc., 68, 241-251, 1982.

Hurwitz, L., E. B. Fabiano, and N. W. Peddie, A model of the geomagnetic field for 1970, J. Geophys. Res., 79, 1716-1717, 1974.

Johnson, I. M. and D. E. SMYLIE, An inverse theory for the calculation of the electrical conductivity of the lower mantle, Geophys. J. R. Astr. Soc., 22, 41-53, 1970.

Kanasewich, E. R., Time Sequence Analysis in Geophysics, 2nd Ed., University of Alberta Press, Edmonton, 1975.

Lowes, F. J., Spatial power spectrum of the main geomagnetic field, and extrapolation to the core, Geophys. J. R. Astr. Soc., 36, 717-730, 1974.

MALIN, S. R. C., Geomagnetic secular variation and its changes, 1942.5 to 1962.5 , Geophys. J. R. Astr. Soc., 17, 415-441, 1969.

Malin, S. R. C. and A. D. Clark, Geomagnetic secular variation, 1962.5 to 1967.5, Geophys J. R. Astr. Soc., 36, $11-20,1974$. 
MCDonald, K. L., Penetration of the geomagnetic secular field through a mantle with variable conductivity, J. Geophys. Res., 62, 117-141, 1957.

MCDonald, K. L., and R. H. Gunst, An analysis of the earth's magnetic field from 1835 to 1965, ESSA Technical Report IER 46-IES 1, U.S. Govt. Printing Office, 1967.

MCQUeEN, H. W. S., Lower mantle conductivity from spatial and temporal properties of the geomagnetic field, M. Sc. Thesis, York University, Canada, 1981.

Peddie, N. W. and E. B. Fabiano, A model of the geomagnetic field for 1975, J. Geophys. Res., 81, 2539-2542, 1976.

Rochester, M. G., and SMYlie, D. E., Geomagnetic core-mantle coupling and the Chandler wobble, Geophys. J. R. Astr. Soc., 10, 289-315, 1965.

SKILES, D. D., On the transmission of the energy in an incompressible magnetohydrodynamic wave into a conducting solid, Phys. Earth Planet. Inter., 5, 99-109, 1972.

SMYLIE, D. E., Magnetic diffusion in a spherically-symmetric conducting mantle, Geophys. J. R. Astr. Soc., 9, $169-184,1965$.

Stacey, F. D., H. W. S. MCQueen, D. E. Smylie, M. G. Rochester, and D. Conley, abstract, EOS, 59, $1027,1978$.

WhaLER, K., Does the whole of the earth's core convect?, Nature, 287, 528-530, 1980.

YukUtake, T., Review of the geomagnetic secular variation on the historical time scale, Phys. Earth Planet. Inter., 20, 83-95, 1979. 